Supporting information for

\title{
Gem-difluorination of Aminoalkynes \\ via Highly Reactive Dicationic Species in Superacid HF-SbF \\ Application to the Efficient Synthesis of Difluorinated Cinchona \\ Alkaloid Derivatives.
}

\author{
Anne-Céline Cantet, Hélène Carreyre, Jean-Pierre Gesson, \\ Marie-Paule Jouannetaud and Brigitte Renoux* \\ Univ Poitiers, CNRS, \\ "Laboratoire "Synthèse et Réactivité des Substances Naturelles" » \\ 40, Avenue du Recteur Pineau, \\ F-86022 POITIERS Cedex \\ e-mail: brigitte.renoux@univ-poitiers.fr
}

Table of contents:

$\begin{array}{ll}\text { General considerations } & \text { S3 }\end{array}$

General procedure for the synthesis of compounds 10-12 S3

2-(4,4-Difluoro-pentyl)-isoindole-1,3-dione $\mathbf{1 0} \quad$ S4

2-(4-Oxo-pentyl)-isoindole-1,3-dione 11

1-(4,4-Difluoro-pentyl)-4-phényl-piperidine $12 \quad$ S4

General procedure for the synthesis of compounds 14-22 S5

2,2-Difluoropropylamine $14 \quad$ S5

(2,2-Difluoropropyl)-phenyl-amine $15 \quad$ S6

Bis-(2,2-Difluoropropyl)-phenyl-amine $\mathbf{1 6}$ S6

1-(2,2-Difluoropropyl)-4-phenyl-piperidine $\mathbf{1 7}$ S6

2-(2,2-Difluoropropyl)-1,2,3,4-tetrahydroisoquinoline $\mathbf{1 8} \quad$ S7 
1-(2,2-Difluoropropyl)-1H-indole 19

2-(2,2-Difluoropropyl)-isoindole-1,3-dione $\mathbf{2 0}$

S8

2-(2-Oxo-propyl)-isoindole-1,3-dione 21

S8

N-(2-Oxopropyl)-N-phenyl-acetamide 22

S9

General procedure for synthesis of compounds 25-28

S9

(3S)-10,10-Difluoro-10,11-dihydro-quinine 25

S9

(3S)-O-Acetyl-3-acetylquinidine $\mathbf{2 6}$

S10

(3R)-O-Acetyl-3-acetylquinidine $\mathbf{2 7}$

S11

(3S)-O-Acetyl-10,10-difluoro-10,11-dihydro-quinidine 28

S11

Copies of spectroscopic data

S13-39

${ }^{1} \mathrm{H},{ }^{19} \mathrm{~F}$ spectra of $\mathbf{1 0}$

$\mathrm{S} 13$

${ }^{1} \mathrm{H}$ spectra of $\mathbf{1 1}$

S14

${ }^{1} \mathrm{H},{ }^{13} \mathrm{C}$ NMR spectra of $\mathbf{1 2}$

S15-16

${ }^{1} \mathrm{H},{ }^{19} \mathrm{~F},{ }^{13} \mathrm{C}$ NMR spectra of $\mathbf{1 4}$

S17-18

${ }^{1} \mathrm{H},{ }^{13} \mathrm{C}$ NMR spectra of $\mathbf{1 5}$

S19-20

${ }^{1} \mathrm{H},{ }^{13} \mathrm{C}$ NMR spectra of $\mathbf{1 6}$

S21

${ }^{1} \mathrm{H},{ }^{19} \mathrm{~F},{ }^{13} \mathrm{C}$ NMR spectra of $\mathbf{1 7}$

S22-24

${ }^{1} \mathrm{H},{ }^{19} \mathrm{~F},{ }^{13} \mathrm{C}$ NMR spectra of $\mathbf{1 8}$

S25-27

${ }^{1} \mathrm{H},{ }^{13} \mathrm{C}$ NMR spectra of 19

S28-29

${ }^{1} \mathrm{H},{ }^{19} \mathrm{~F},{ }^{13} \mathrm{C}$ NMR spectra of $\mathbf{2 0}$

S30-32

${ }^{1} \mathrm{H},{ }^{13} \mathrm{C}$ NMR spectra of $\mathbf{2 1}$

S33-34

${ }^{1} \mathrm{H},{ }^{19} \mathrm{~F},{ }^{13} \mathrm{C}$ NMR spectra of 25

S35-36

${ }^{1} \mathrm{H},{ }^{13} \mathrm{C}$ NMR spectra of 26

${ }^{1} \mathrm{H},{ }^{13} \mathrm{C}$ NMR spectra of 27

S38

${ }^{1} \mathrm{H},{ }^{13} \mathrm{C}$ NMR spectra of $\mathbf{2 8}$

S39 


\section{EXPERIMENTAL SECTION}

\section{General considerations}

Handling of hydrogen fluoride and antimony pentafluoride must be done by experienced chemists with all the necessary safety arrangements in place.

Reactions performed in superacid were carried out in a sealed Teflon ${ }^{\circledR}$ flask with a magnetic stirrer. No further precautions have to be taken to prevent ingress of moisture (test reaction in anhydrous conditions leads to the same results as expected).

Yields refer to isolated pure products.

${ }^{1} \mathrm{H},{ }^{13} \mathrm{C},{ }^{19} \mathrm{~F}$ NMR were recorded on a $300 \mathrm{MHz}$ spectrometer using $\mathrm{CDCl}_{3}$ as solvent and TMS as internal standard. NMR multiplicities are reported using the following abbreviations (s: singlet, d: doublet, t: triplet, q: quartet, m: multiplet, br: broad, $J$ : coupling constants in Hertz).

Melting points were determined in a capillary tube and are uncorrected.

The reactions were monitored by thin layer chromatography carried out on $0.2 \mathrm{~mm}$ silica gel $60 \AA$ plates.

All separations were done under flash-chromatography conditions on silica gel $(15-40 \mu \mathrm{m})$.

Optical rotations were measured on a polarimeter.

\section{General procedure for the synthesis of compounds 10-12}

In a typical experiment, to a stirred solution of $\mathrm{HF}(5 \mathrm{~mL} / \mathrm{mmol})$ at $0^{\circ} \mathrm{C}$ in a Teflon ${ }^{\circledR}$ reactor was added the substrate. The reaction was kept at $0^{\circ} \mathrm{C}$ for $5 \mathrm{~h}$. The mixture was then neutralized with $\mathrm{Na}_{2} \mathrm{CO}_{3}-\mathrm{H}_{2} \mathrm{O}$-ice. After extraction with dichloromethane, the combined organic phases were dried $\left(\mathrm{MgSO}_{4}\right)$ and concentrated in vacuo. Purification of the residue by chromatography gave the pure derivatives. 


\section{2-(4,4-Difluoro-pentyl)-isoindole-1,3-dione 10}

Starting material: imide $\mathbf{1}$ (1.4 mmol), Flash chromatography over $\mathrm{SiO}_{2}: \mathrm{CH}_{2} \mathrm{Cl}_{2}$, Yield for $\mathbf{1 0}$ (190 mg, 53\%, white powder) and for 11 (51 mg, 17\%, white powder). The spectral data for 10 were as follows: ${ }^{1} \mathrm{H}$ NMR (300 MHz, $\left.\mathrm{CDCl}_{3}\right) \delta(\mathrm{ppm}):$ 7.88-7.83 (m, 2H, H-4 and H-7), 7.76-7.70 (m, 2H, H-5 and H-6), 3.74 (t, $\left.J=6.6 \mathrm{~Hz}, 2 \mathrm{H}, \mathrm{H}-1^{\prime}\right), 1.93-1.87$ (m, 4H, H-2' and H-3'), $1.59\left(\mathrm{t},{ }^{1} J_{H F}=18.4 \mathrm{~Hz}, 3 \mathrm{H}, \mathrm{H}-5\right.$ '). ${ }^{13} \mathrm{C} \mathrm{NMR}\left(75 \mathrm{MHz}, \mathrm{CDCl}_{3}\right) \delta(\mathrm{ppm}): 168.3(\mathrm{C}-1$ and C-3), 134.0 (C-5 and C-6), 132.0 (C-3a and C-7a), 123.7 (t, ${ }^{1} J_{C F}=238 \mathrm{~Hz}, \mathrm{C}-4$ '), 123.3 (C-4 and C-7), $37.4\left(\mathrm{C}-1^{\prime}\right), 35.4\left(\mathrm{t},{ }^{2} J_{C F}=26.4 \mathrm{~Hz}, \mathrm{C}-3\right.$ ') $, 23.4\left(\mathrm{t},{ }^{2} J_{C F}=27.2 \mathrm{~Hz}, \mathrm{C}-5^{\prime}\right), 22.1$ $\left(\mathrm{C}-2\right.$ '). ${ }^{19} \mathrm{~F}$ NMR $\left(282 \mathrm{MHz}, \mathrm{CDCl}_{3}\right) \delta(\mathrm{ppm})$ : -91.4 (m). HRMS Calcd. for $\mathrm{C}_{13} \mathrm{H}_{13} \mathrm{NO}_{2} \mathrm{~F}_{2}=$ 253.09144, Found: 253.0908. m.p. $76^{\circ} \mathrm{C}$.

\section{2-(4-Oxo-pentyl)-isoindole-1,3-dione 11}

The spectral data for 11 were as follows: ${ }^{1} \mathrm{H}$ NMR $\left(300 \mathrm{MHz}, \mathrm{CDCl}_{3}\right) \delta(\mathrm{ppm})$ : 7.87-7.82 (m, 2H, H-4 and H-7), 7.75-7.67 (m, 2H, H-5 and H-6), 3.71 (t, $J=6.7 \mathrm{~Hz}, 2 \mathrm{H}, \mathrm{H}-1$ '), 2.51 (t, $J=$ $\left.7.2 \mathrm{~Hz}, 2 \mathrm{H}, \mathrm{H}-3^{\prime}\right), 2.14$ (s, 3H, H-5'), 1.94 (m, 2H, H-2'). ${ }^{13} \mathrm{C}$ NMR (75 MHz, $\left.\mathrm{CDCl}_{3}\right) \delta$ (ppm): 207.5 (C-4'), 168.5 (C-1 and C-3), 134.0 (C-5 and C-6), 132.1 (C-3a and C-7a), 123.3 (C-4 and C-7), 40.6 (C-3'), 37.2 (C-1'), 29.9 (C-5'), 22.7 (C-2'). HRMS Calcd. for $\mathrm{C}_{13} \mathrm{H}_{13} \mathrm{NO}_{3}=231.08954$, Found: 231.0911.m.p. $70^{\circ} \mathrm{C}$.

\section{1-(4,4-Difluoro-pentyl)-4-phényl-piperidine 12}

Starting material: amine $2(0.88 \mathrm{mmol})$, Flash chromatography over $\mathrm{SiO}_{2}: \mathrm{CH}_{2} \mathrm{Cl}_{2}$ /acetone: 90/10), Yield for $\mathbf{1 2}$ (200 $\mathrm{mg}, 85 \%$, white powder). The spectral data for $\mathbf{1 2}$ were as follows:

${ }^{1} \mathrm{H}$ NMR (300 MHz, $\mathrm{CDCl}_{3}$ ) $\delta$ (ppm): 7.33-7.20 (m, 5H, H-Ar), 3.05 (d, $J=11.5 \mathrm{~Hz}, 2 \mathrm{H}, \mathrm{H}-$ 2a and H-6a), 2.55-2.43 (m, 1H, H-4), $2.41\left(\mathrm{t}, J=7.5 \mathrm{~Hz}, 2 \mathrm{H}, \mathrm{H}-1^{\prime}\right), 2.07(\mathrm{td}, J=11.1 \mathrm{~Hz}, J=$ 3.7 Hz, 2H, H-2b and H-6b), 1.94-1.69 (m, 8H, H-3, H-5, H-2' and H-3'), 1.61 (t, $J=18.4$ Hz, 
3H, H-5'). ${ }^{13} \mathrm{C} \mathrm{NMR}\left(75 \mathrm{MHz}, \mathrm{CDCl}_{3}\right) \delta(\mathrm{ppm}): 146.4$ (C-7), 128.8 (C-9 and C-11), 127.2 (C8 and C-12), 126.5 (C-10), $127.4\left(\mathrm{t},{ }^{1} J_{C F}=238 \mathrm{~Hz}, \mathrm{C}-4\right.$ '), 58.7 (C-1'), 54.7 (C-2 and C-6), $43.1(\mathrm{C}-4), 36.3\left(\mathrm{t},{ }^{2} J_{C F}=26 \mathrm{~Hz}, \mathrm{C}-3\right.$ '), 33.9 (C-3 and C-5), 23.7(t, ${ }^{2} J_{C F}=28 \mathrm{~Hz}, \mathrm{C}-5$ '), 20.8 $\left(\mathrm{t},{ }^{3} J_{C F}=4.6 \mathrm{~Hz}, \mathrm{C}-2{ }^{\prime}\right) .{ }^{19} \mathrm{~F}$ NMR $\left(282 \mathrm{MHz}, \mathrm{CDCl}_{3}\right) \delta(\mathrm{ppm}):-90.9$ (m). HRMS Calcd. for $\mathrm{C}_{16} \mathrm{H}_{23} \mathrm{NF}_{2}=267.17986$, Found: 267.1790.

\section{General procedure for the synthesis of compounds 14-22}

In a typical experiment, to a stirred solution of $\mathrm{HF}(5 \mathrm{~mL})$ and $\mathrm{SbF}_{5}(2.5 \mathrm{~mL})$ at low temperature in a Teflon ${ }^{\circledR}$ reactor was added the substrate $(1.8 \mathrm{mmol})$. The reaction was kept at a given temperature for 2-15 min. according to the substrate. The mixture was then neutralized with $\mathrm{Na}_{2} \mathrm{CO}_{3}-\mathrm{H}_{2} \mathrm{O}$-ice. After extraction with dichloromethane, the combined organic phases were dried $\left(\mathrm{MgSO}_{4}\right)$ and concentrated in vacuo. Purification of the residue by chromatography gave the pure derivatives.

\section{2,2-Difluoropropylamine 14}

Starting material: amine $13(10 \mathrm{mmol})$, Reaction time: $2 \mathrm{~min}$, Temperature: $-40^{\circ} \mathrm{C}$. After usual extraction, few drops of a solution of $\mathrm{HCl} / \mathrm{MeOH}$ were added to the combined organic phases. Evaporation to dryness afforded $14(220 \mathrm{mg}, 18 \%)$ as a beige powder. The spectral data for 14 were as follows: ${ }^{1} \mathrm{H}$ NMR $\left(300 \mathrm{MHz}, \mathrm{D}_{2} \mathrm{O}\right) \delta(\mathrm{ppm}): 3.40(\mathrm{t}, J=15.3 \mathrm{~Hz}, 2 \mathrm{H}, \mathrm{H}-$ 1), $1.80(\mathrm{t}, J=19.3 \mathrm{~Hz}, 3 \mathrm{H}, \mathrm{H}-3) .{ }^{13} \mathrm{C} \mathrm{NMR}\left(75 \mathrm{MHz}, \mathrm{D}_{2} \mathrm{O}\right) \delta(\mathrm{ppm}): 121.2\left(\mathrm{t},{ }^{l} J_{C F}=239 \mathrm{~Hz}\right.$, $\mathrm{C}-2), 44.2\left(\mathrm{t},{ }^{2} J_{C F}=26 \mathrm{~Hz}, \mathrm{C}-1\right), 21.2\left(\mathrm{t},{ }^{2} J_{C F}=25 \mathrm{~Hz}, \mathrm{C}-3\right) .{ }^{19} \mathrm{~F}$ NMR $\left(282 \mathrm{MHz}, \mathrm{D}_{2} \mathrm{O}\right) \delta$ (ppm): -98.2 (m). HRMS Calcd. for $\mathrm{C}_{3} \mathrm{H}_{7} \mathrm{NF}_{2}=95.05466$, Found: 95.0555. 


\section{(2,2-Difluoropropyl)-phenyl-amine 15}

Starting material: amine 3 (4.6 mmol), Reaction time: $15 \mathrm{~min}$, Temperature: $-60^{\circ} \mathrm{C}$, Flash chromatography over $\mathrm{SiO}_{2}$ : PE/EtOAc: 99/1 and 97/3, Yield for 15 (665 mg, 85\%, yellow oil). The spectral data for 15 were as follows: ${ }^{1} \mathrm{H}$ NMR (300 MHz, $\left.\mathrm{CDCl}_{3}\right) \delta(\mathrm{ppm})$ : 7.24-7.16 (m, 2H, H-3 and H-5), 6.76 (m, 1H, H-4), 6.65 (m, 2H, H-2 and H-6), 3.87 (br s, 1H, N- $)$, 3.57-3.43 (td, $J=13 \mathrm{~Hz}$ and $\left.J=6.4 \mathrm{~Hz}, 2 \mathrm{H}, \mathrm{H}-1^{\prime}\right), 1.67$ (t, $\left.J=18.6 \mathrm{~Hz}, 3 \mathrm{H}, \mathrm{H}-3^{\prime}\right) .{ }^{13} \mathrm{C}$ NMR $\left(75 \mathrm{MHz}, \mathrm{CDCl}_{3}\right) \delta(\mathrm{ppm}): 147.6(\mathrm{C}-1), 129.8\left(\mathrm{C}-3\right.$ and C-5), $123.5\left(\mathrm{t},{ }^{1} J_{C F}=241 \mathrm{~Hz}, \mathrm{C}-2{ }^{\prime}\right)$, $118.8(\mathrm{C}-4), 113.5\left(\mathrm{C}-2\right.$ and C-6), $49.9\left(\mathrm{t},{ }^{2} J_{C F}=30 \mathrm{~Hz}, \mathrm{C}-1\right.$ ' $), 22.1\left(\mathrm{t},{ }^{2} J_{C F}=27 \mathrm{~Hz}, \mathrm{C}-3^{\prime}\right)$. ${ }^{19} \mathrm{~F}$ NMR (282 MHz, $\left.\mathrm{CDCl}_{3}\right) \delta(\mathrm{ppm})$ : -96.7 (m). HRMS Calcd. for $\mathrm{C}_{9} \mathrm{H}_{11} \mathrm{NF}_{2}=171.08596$, Found: 171.0863 .

\section{Bis-(2,2-Difluoropropyl)-phenyl-amine 16}

Starting material: amine 4 (2.4 mmol), Reaction time: $10 \mathrm{~min}$, Temperature: $-60^{\circ} \mathrm{C}$, Flash chromatography over $\mathrm{SiO}_{2}$ : PE/EtOAc: 99/1 and 97/3, Yield for 16 (350 mg, 60\%, colourless oil). The spectral data for 16 were as follows: ${ }^{1} \mathrm{H}$ NMR (300 MHz, $\left.\mathrm{CDCl}_{3}\right) \delta(\mathrm{ppm}):$ 7.26-7.21 (m, 2H, H-3 and H-5), 6.89 (m, 2H, H-2 and H-6), $6.80(\mathrm{~m}, 1 \mathrm{H}, \mathrm{H}-4), 3.94(\mathrm{t}, J=13.2 \mathrm{~Hz}$, 4H, H-1'), 1.59 (t, $J=18.9 \mathrm{~Hz}, 6 \mathrm{H}, \mathrm{H}-3$ '). ${ }^{13} \mathrm{C} \mathrm{NMR}\left(75 \mathrm{MHz}, \mathrm{CDCl}_{3}\right) \delta(\mathrm{ppm}): 147.9$ (C-1), $129.3\left(\mathrm{C}-3\right.$ and C-5), $122.1\left(\mathrm{t},{ }^{1} J_{C F}=242 \mathrm{~Hz}, \mathrm{C}-2\right.$ ') $117.2(\mathrm{C}-4), 114.2$ (C-2 and C-6), $52.2(\mathrm{t}$, $\left.{ }^{2} J_{C F}=29 \mathrm{~Hz}, \mathrm{C}-1^{\prime}\right), 21.7\left(\mathrm{t},{ }^{2} J_{C F}=27 \mathrm{~Hz}, \mathrm{C}-3{ }^{\prime}\right) .{ }^{19} \mathrm{~F}$ NMR $\left(282 \mathrm{MHz}, \mathrm{CDCl}_{3}\right) \delta(\mathrm{ppm}):-92.3$ (m). HRMS Calcd. for $\mathrm{C}_{12} \mathrm{H}_{15} \mathrm{NF}_{4}$ : $[\mathrm{M}+]=$.249.11406 , Found: 249.1132; Calcd. for $\mathrm{C}_{10} \mathrm{H}_{15} \mathrm{NF}_{4}:\left[\mathrm{M}-. \mathrm{CF}_{2} \mathrm{CH}_{3}\right]=184.09378$, Found: 184.0928 .

\section{1-(2,2-Difluoropropyl)-4-phenyl-piperidine 17}

Starting material: amine $5(1.5 \mathrm{mmol})$, Reaction time: $10 \mathrm{~min}$, Temperature: $-50^{\circ} \mathrm{C}$, Flash chromatography over $\mathrm{SiO}_{2}: \mathrm{CH}_{2} \mathrm{Cl}_{2}$ /Acetone: 98/2 and 95/5, Yield for 17 (315 mg, 87\%, 
brown powder). The spectral data for $\mathbf{1 7}$ were as follows: ${ }^{1} \mathrm{H}$ NMR $\left(300 \mathrm{MHz}, \mathrm{CDCl}_{3}\right) \delta$ (ppm): 7.33-7.17 (m, 5H, H-Ar), 3.05 (d, $J=10.8 \mathrm{~Hz}, 2 \mathrm{H}, \mathrm{H}-2 \mathrm{a}$ and H-6a), 2.70 (t, $J=13.8$ Hz, 2H, H-1'), 2.45 (m, 1H, H-4), 2.30 (dd, $J=11.1 \mathrm{~Hz}, J=3.6 \mathrm{~Hz}, 2 \mathrm{H}, \mathrm{H}-2 \mathrm{~b}$ and H-6b), $1.79(\mathrm{~m}, 4 \mathrm{H}, \mathrm{H}-3$ and $\mathrm{H}-5), 1.66\left(\mathrm{t}, J=18.7 \mathrm{~Hz}, 3 \mathrm{H}, \mathrm{H}-3{ }^{\prime}\right) .{ }^{13} \mathrm{C}$ NMR $\left(75 \mathrm{MHz}, \mathrm{CDCl}_{3}\right) \delta$ (ppm): 146.3 (C-7), 128.4 (C-9 and C-11), 126.8 (C-10), 126.1 (C-8 and C-12), $124.4\left(\mathrm{t},{ }^{1} J_{C F}\right.$ $=239 \mathrm{~Hz}, \mathrm{C}-2$ '), $63.0\left(\mathrm{t},{ }^{2} J_{C F}=28 \mathrm{~Hz}, \mathrm{C}-1\right.$ '), 55.5 (C-2 and C-6), $42.2(\mathrm{C}-4), 33.6(\mathrm{C}-3$ and C5), $22.0\left(\mathrm{t},{ }^{2} J_{C F}=27 \mathrm{~Hz}, \mathrm{C}-3{ }^{\prime}\right) .{ }^{19} \mathrm{~F}$ NMR $\left(282 \mathrm{MHz}, \mathrm{CDCl}_{3}\right) \delta(\mathrm{ppm}):-92.3(\mathrm{~m})$. HRMS Calcd. for $\mathrm{C}_{14} \mathrm{H}_{19} \mathrm{NF}_{2}=239.14856$, Found: 239.1497 . m.p. $36^{\circ} \mathrm{C}$.

\section{2-(2,2-Difluoropropyl)-1,2,3,4-tetrahydroisoquinoline 18}

Starting material: amine $6(1.7 \mathrm{mmol})$, Reaction time: $10 \mathrm{~min}$, Temperature: $-60^{\circ} \mathrm{C}$, Flash chromatography over $\mathrm{SiO}_{2}: \mathrm{CH}_{2} \mathrm{Cl}_{2}$, Yield for 18 (220 mg, 61\%, orange oil). The spectral data for 18 were as follows: ${ }^{1} \mathrm{H}$ NMR $\left(300 \mathrm{MHz}, \mathrm{CDCl}_{3}\right) \delta$ (ppm): 7.15-7.10 (m, 3H, H-Ar), 7.01 (m, 1H, H-Ar), 3.79 (s, 2H, H-1), 2.89 (s, 4H, H-3 and H-4), 2.85 (t, J=13.4 Hz, 2H, H-1'), $1.68\left(\mathrm{t}, J=18.8 \mathrm{~Hz}, 3 \mathrm{H}, \mathrm{H}-3\right.$ '). ${ }^{13} \mathrm{C}$ NMR $\left(75 \mathrm{MHz}, \mathrm{CDCl}_{3}\right) \delta(\mathrm{ppm}): 134.6(\mathrm{C}-8 \mathrm{a}), 134.0(\mathrm{C}-$ 4a), 128.7 (C-8), 126.5 (C-5), 126.2 (C-6), 125.7 (C-7), 124.4 (t, ${ }^{1} J_{C F}=239 \mathrm{~Hz}, \mathrm{C}-2$ '), 62.3 (t, $\left.{ }^{2} J_{C F}=28 \mathrm{~Hz}, \mathrm{C}-1^{\prime}\right), 56.8(\mathrm{C}-3), 51.9(\mathrm{C}-1), 29.0(\mathrm{C}-4), 21.8\left(\mathrm{t},{ }^{2} J_{C F}=27 \mathrm{~Hz}, \mathrm{C}-3{ }^{\prime}\right) .{ }^{19} \mathrm{~F}$ NMR $\left(282 \mathrm{MHz}, \mathrm{CDCl}_{3}\right) \delta(\mathrm{ppm})$ : -92.1 (m). HRMS Calcd. for $\mathrm{C}_{12} \mathrm{H}_{15} \mathrm{NF}_{2}=211.11726$, Found: 211.1173 .

\section{1-(2,2-Difluoropropyl)-1H-indole 19}

Starting material: amine $7(1.8 \mathrm{mmol})$, Reaction time: $10 \mathrm{~min}$, Temperature: $-50^{\circ} \mathrm{C}$, Flash chromatography over $\mathrm{SiO}_{2}$ : PE/EtOAc: 95/5, Yield for 19 (280 mg, 74\%, yellow oil). The spectral data for 19 were as follows: ${ }^{1} \mathrm{H}$ NMR $\left(300 \mathrm{MHz}, \mathrm{CDCl}_{3}\right) \delta(\mathrm{ppm}): 7.63(\mathrm{~d}, \mathrm{~J}=7.3 \mathrm{~Hz}$, 1H, H-4), 7.34 (d, $J=8.1 \mathrm{~Hz}, 1 \mathrm{H}, \mathrm{H}-7), 7.26-7.13$ (m, 2H, H-5 and H-6), 7.10 (d, $J=3.2 \mathrm{~Hz}$, 
1H, H-2), 6.56 (d, $J=3.2 \mathrm{~Hz}, 1 \mathrm{H}, \mathrm{H}-3), 4.39$ (t, $\left.J=13.0 \mathrm{~Hz}, 2 \mathrm{H}, \mathrm{H}-1^{\prime}\right), 1.51$ (t, $J=18.5 \mathrm{~Hz}$, 3H, H-3'). ${ }^{13} \mathrm{C}$ NMR (75 MHz, $\left.\mathrm{CDCl}_{3}\right) \delta(\mathrm{ppm}): 136.6$ (C-7a), 128.6 (C-3a), 128.5 (C-2), $122.2(\mathrm{C}-6), 122.2\left(\mathrm{t},{ }^{1} J_{C F}=242 \mathrm{~Hz}, \mathrm{C}-2\right.$ '), 121.1 (C-4), 119.9 (C-5), 109.3 (C-7), 102.9 (C3), $51.2\left(\mathrm{t},{ }^{2} J_{C F}=33 \mathrm{~Hz}, \mathrm{C}-1^{\prime}\right), 21.1\left(\mathrm{t},{ }^{2} J_{C F}=26 \mathrm{~Hz}, \mathrm{C}-3{ }^{\prime}\right) .{ }^{19} \mathrm{~F}$ NMR $\left(282 \mathrm{MHz}, \mathrm{CDCl}_{3}\right) \delta$ (ppm): -94.4 (m). HRMS Calcd. for $\mathrm{C}_{12} \mathrm{H}_{15} \mathrm{NF}_{4}=249.11406$, Found: 249.1132.

\section{2-(2,2-Difluoropropyl)-isoindole-1,3-dione 20}

Starting material: imide $8(2.7 \mathrm{mmol})$, Reaction time: $10 \mathrm{~min}$, Temperature: $-50^{\circ} \mathrm{C}$, Flash chromatography over $\mathrm{SiO}_{2}$ : PE/EtOAc: 90/10, Yield for $\mathbf{2 0}$ (410 mg, 67\%, white powder) and for 21 (95 mg, 17\%, white powder). The spectral data for 20 were as follows: ${ }^{1} \mathrm{H}$ NMR (300 $\left.\mathrm{MHz}, \mathrm{CDCl}_{3}\right) \delta(\mathrm{ppm}): 7.89$ (m, 2H, H-4 and H-7), 7.77 (m, 2H, H-5 and H-6), 4.07 (t, 2H, ${ }^{1} J_{H F}=13 \mathrm{~Hz}, \mathrm{H}-1$ ') $1.69\left(\mathrm{t}, 3 \mathrm{H},{ }^{1} J_{H F}=13 \mathrm{~Hz}, \mathrm{H}-3\right.$ '). ${ }^{13} \mathrm{C} \mathrm{NMR}\left(75 \mathrm{MHz}, \mathrm{CDCl}_{3}\right) \delta(\mathrm{ppm})$ : 167.5 (C-1 and C-3), 134.4 (C-5 and C-6), 131.8 (C-3a and C-7a), 123.7 (C-4 and C-7), 121.2 $\left(\mathrm{t},{ }^{1} J_{C F}=226 \mathrm{~Hz}, \mathrm{C}-2^{\prime}\right), 42.6\left(\mathrm{t},{ }^{2} J_{C F}=32 \mathrm{~Hz}, \mathrm{C}-1\right.$ '), $31.2\left(\mathrm{t},{ }^{2} J_{C F}=26 \mathrm{~Hz}, \mathrm{C}-3^{\prime}\right) .{ }^{19} \mathrm{~F}$ NMR $\left(282 \mathrm{MHz}, \mathrm{CDCl}_{3}\right) \delta(\mathrm{ppm}):-95.2(\mathrm{~m})$. HRMS Calcd. for $\mathrm{C}_{11} \mathrm{H}_{9} \mathrm{NO}_{2} \mathrm{~F}_{2}=225.06014$, Found: 225.0598. m.p. $112^{\circ} \mathrm{C}$.

\section{2-(2-Oxo-propyl)-isoindole-1,3-dione 21}

The spectral data for 21 were as follows: ${ }^{1} \mathrm{H}$ NMR $\left(300 \mathrm{MHz}, \mathrm{CDCl}_{3}\right) \delta(\mathrm{ppm}): 7.87(\mathrm{~m}$, 2H, H-4 and H-7), 7.75 (m, 2H, H-5 and H-6), 4.45 (s, 2H, H-1'), 2.28 (s, 3H, H-3'). ${ }^{13} \mathrm{C}$ NMR (75 MHz, $\left.\mathrm{CDCl}_{3}\right) \delta(\mathrm{ppm}): 199.2\left(\mathrm{C}-2^{\prime}\right.$ ), 167.6 (C-1 and C-3), 134.2 (C-5 and C-6), 132.0 (C-3a and C-7a), 123.5 (C-4 and C-7), 47.1 (C-1'), 27.0 (C-3'). HRMS Calcd. for $\mathrm{C}_{11} \mathrm{H}_{9} \mathrm{NO}_{3}=203.05824$, Found: 203.0577 . m.p. $112^{\circ} \mathrm{C}$. 


\section{N-(2-Oxopropyl)-N-phenyl-acetamide 22}

Starting material: amide 9 (3.5 mmol), Reaction time: $10 \mathrm{~min}$, Temperature: $-60^{\circ} \mathrm{C}$, Flash chromatography over $\mathrm{SiO}_{2}$ : PE/EtOAc: 50/50, Yield for 22 (300 mg, 45\%, yellow powder). The spectral data for 22 were as follows: ${ }^{1} \mathrm{H}$ NMR $\left(300 \mathrm{MHz}, \mathrm{CDCl}_{3}\right) \delta(\mathrm{ppm})$ : 7.40-7.30 (m, 5H, H-Ar), 4.44 (s, 2H, H-1'), 2.16 (s, 2H, H-3'), 1.92 (s, 3H, $\left.\underline{\mathrm{H}}_{3}-\mathrm{C}(\mathrm{O}) \mathrm{N}\right) .{ }^{13} \mathrm{C}$ NMR (75 $\left.\mathrm{MHz}, \mathrm{CDCl}_{3}\right) \delta$ (ppm): 202.8 (C-2'), $171.1(\mathrm{C}=\mathrm{O}$ amide), 143.8 (C-1), 130.1 (C-3 and C-5), 128.5 (C-4), 128.2 (C-2 and C-6), 59.7 (C-1'), $27.5\left(\mathrm{C}-3{ }^{\prime}\right), 22.5\left(\underline{\mathrm{CH}}_{3}-\mathrm{C}(\mathrm{O}) \mathrm{N}\right)$. m.p. $121^{\circ} \mathrm{C}$.

\section{General procedure for synthesis of compounds 25, 26, 27 and 28}

To a mixture of $\mathrm{HF} / \mathrm{SbF}_{5}$ maintained at low temperature in a Teflon ${ }^{\circledR}$ flask, was added quinine or quinidine derivatives. The reaction mixture was magnetically stirred at the same temperature for few minutes. The reaction mixture was then neutralized with water/ice (300 $\mathrm{mL})$ and $\mathrm{Na}_{2} \mathrm{CO}_{3}(100 \mathrm{~g})$, and worked-up by usual manner. The products were isolated by column chromatography over $\mathrm{SiO}_{2}$.

\section{(3S)-10,10-Difluoro-10,11-dihydro-quinine 25}

Starting material $23(0.94 \mathrm{mmol}) ; \mathrm{HF} / \mathrm{SbF}_{5} \mathrm{v} / \mathrm{v}: 6 / 3 \mathrm{~mL}$; Temperature: $-40^{\circ} \mathrm{C}$; Reaction time: 10 min., Flash chromatography over $\mathrm{SiO}_{2}: \mathrm{CH}_{2} \mathrm{Cl}_{2} / \mathrm{MeOH} / \mathrm{NH}_{4} \mathrm{OH}$ : 95/4/1, Yield for 25: (255 mg, 74\%, white powder). The spectral data for 25 were as follows: ${ }^{1} \mathrm{H}$ NMR (300 $\left.\mathrm{MHz}, \mathrm{CDCl}_{3}\right) \delta(\mathrm{ppm}): 8.47\left(\mathrm{~d}, J=4.5 \mathrm{~Hz}, 1 \mathrm{H}, \mathrm{H}-2^{\prime}\right), 7.88\left(\mathrm{~d}, J=9.2 \mathrm{~Hz}, 1 \mathrm{H}, \mathrm{H}-8^{\prime}\right), 7.40(\mathrm{~d}$, $\left.J=4.5 \mathrm{~Hz}, 1 \mathrm{H}, \mathrm{H}-3^{\prime}\right), 7.29-7.20$ (m, 2H, H-5' and H-7'), 5.43 (d, $\left.J=4.3 \mathrm{~Hz}, 1 \mathrm{H}, \mathrm{H}-9\right), 3.89$ (s, 3H, OC$\left.{ }_{3}\right), 3.45$ (m, 1H, H-6a), 3.25 (m, 1H, H-8), 2.93 (d, J=8.0 Hz, 2H, H-2), 2.55 (m, 1H, H-6b), 1.99 (m, 1H, H-4), 1.88 (m, 1H, H-3), 1.68 (m, 2H, H-5a and H-7a), 1.50 (m and t, 
$J=18.6 \mathrm{~Hz}, 5 \mathrm{H}, \mathrm{H}-5 \mathrm{~b}, \mathrm{H}-7 \mathrm{~b}$ and H-11). ${ }^{13} \mathrm{C}$ NMR $\left(75 \mathrm{MHz}, \mathrm{CDCl}_{3}\right) \delta(\mathrm{ppm}): 158.0$ (C-6'), 148.9 (C-4'), 147.4 (C-2'), 144.2 (C-9'), 131.3 (C-8'), 127.0 (C-10'), 125.4 (t, ${ }^{1} J_{C F}=240 \mathrm{~Hz}$, C-10), 121.7 (C-7'), 119.0 (C-3'), 102.0 (C-5'), 72.2 (C-9), 60.1 (C-8), 56.0 (OCH$\left.{ }_{3}\right), 51.7$ (C2), $43.4(\mathrm{C}-6), 43.1\left(\mathrm{t},{ }^{2} J_{C F}=23.4 \mathrm{~Hz}, \mathrm{C}-3\right), 28.8(\mathrm{C}-5), 23.4\left(\mathrm{t},{ }^{3} J_{C F}=5.2 \mathrm{~Hz}, \mathrm{C}-4\right), 23.3$ (t, $\left.{ }^{2} J_{C F}=28.1 \mathrm{~Hz}, \mathrm{C}-11\right), 22,9(\mathrm{C}-7) .{ }^{19} \mathrm{~F} \mathrm{NMR}\left(282 \mathrm{MHz}, \mathrm{CDCl}_{3}\right) \delta(\mathrm{ppm}):-94.5$ (dquint, $J=$ $262 \mathrm{~Hz}, J=18.9 \mathrm{~Hz})$ and $-97.2(\mathrm{dm}, J=262 \mathrm{~Hz})$. m.p. $186^{\circ} \mathrm{C} .[\alpha]_{\mathrm{D}}:-84^{\circ}\left(c 0.40, \mathrm{CHCl}_{3}\right.$, $\left.20^{\circ} \mathrm{C}\right)$.

\section{(3S)-O-Acetyl-3-acetylquinidine 26 and (3R)-O-Acetyl-3-acetylquinidine $27^{1}$}

After reaction of $\mathbf{2 4 a}(470 \mathrm{mg}, 1.46 \mathrm{mmol})$, following the general procedure $\left(\mathrm{HF} / \mathrm{SbF}_{5}: 8 / 4\right.$ $\mathrm{mL},-25^{\circ} \mathrm{C}, 15 \mathrm{~min}$.), two inseparable methylketones were obtained. Acetylation of the mixture in presence of acetic anhydride $(0.41 \mathrm{~mL})$ allows a separation after column chromatography (EtOAc/Et 2 NH: 98/2). Successively, 27 (55 mg, 10\%, yellowish powder) and 26 (307 mg, 55\%, yellowish powder) were isolated.

\section{(3S)-O-Acetyl-3-acetylquinidine 26}

${ }^{1} \mathrm{H}$ NMR (300 MHz, $\left.\mathrm{CDCl}_{3}\right) \delta(\mathrm{ppm}): 8.72$ (d, $J=4.5 \mathrm{~Hz}, 1 \mathrm{H}, \mathrm{H}-2$ '), 8.00 (d, $J=9.2 \mathrm{~Hz}$, 1H, H-8'), 7.61 (d, $J=2.7 \mathrm{~Hz}, 1 \mathrm{H}, \mathrm{H}-5$ ') 7.36 (dd, $\left.J=9.2 \mathrm{~Hz}, J=2.7 \mathrm{~Hz}, 1 \mathrm{H}, \mathrm{H}-7^{\prime}\right), 7.32$ (d, $J=4.5 \mathrm{~Hz}, 2 \mathrm{H}, \mathrm{H}-3$ '), 6.57 (d, $J=7.3 \mathrm{~Hz}, 1 \mathrm{H}, \mathrm{H}-9), 4.01$ (s, 3H, OC$\left.{ }_{3}\right), 3.62(\mathrm{ddd}, J=12.8$ $\mathrm{Hz}, J=5.7 \mathrm{~Hz}, J=2.3 \mathrm{~Hz}, 1 \mathrm{H}, \mathrm{H}-2 \mathrm{a}), 3.23$ (m, 1H, H-8), 2.82 (m, 1H, H-6a), 2.71 (m, 1H, H-6b), 2.60 (m, 2H, H-2b and H-3), 2.30 (m, 1H, H-4), 2.21 (s, 3H, H-11), 2.13 (s, 3H, H-13),

\footnotetext{
${ }^{1}$ Compounds 26 and 27 only differ in the C3 configuration.. In the ${ }^{13} \mathrm{C}$ NMR spectrum of ketone $26 \mathrm{C} 7$ carbon (24.9 ppm) is more shielded than C5 carbon (26.9 ppm), whereas the reverse was observed for 27. A similar effect was observed with methylketone derivatives of 9-epiquinidine (Debarge, S.; Violeau, B.; Jouannetaud, M. P.; Jacquesy, J. C.; Cousson, A. Tetrahedron 2006, 62, 662-671.)
} 
1.64-1.55 (m, 4H, H-5 and H-7). ${ }^{13} \mathrm{C}$ NMR (75 MHz, $\left.\mathrm{CDCl}_{3}\right) \delta(\mathrm{ppm}): 209.1$ (C-10), 169.9 (C-12), 158.0 (C-6'), 147.4 (C-2'), 144.7 (C-10'), 144.1 (C-4'), 131.7 (C-8'), 127.2 (C-9'), 122.1 (C-7'), 118.5 (C-3'), 101.5 (C-5'), 73.6 (C-9), 59.2 (C-8), $\left.55.7\left(\mathrm{OC}_{3}\right)_{3}\right), 50.0$ (C-6), 49.6 (C-3), 43.9 (C-2), 29.0 (C-11), 26.2 (C-5), 25.4 (C-4), 24.9 (C-7), 21.0 (C-13). HRMS $\left(\mathrm{C}_{22} \mathrm{H}_{26} \mathrm{~N}_{2} \mathrm{O}_{4}\right)$ : Calcd. 382.18926, Found 382.1869. m.p. $42^{\circ} \mathrm{C} .[\alpha]_{\mathrm{D}}: 41^{\circ}$ (c 1.0, $\mathrm{CHCl}_{3}$, $\left.20^{\circ} \mathrm{C}\right)$

\section{(3R)-O-Acetyl-3-acetylquinidine 27}

${ }^{1} \mathrm{H}$ NMR (300 MHz, $\left.\mathrm{CDCl}_{3}\right) \delta(\mathrm{ppm}): 8.74(\mathrm{~d}, J=4.5 \mathrm{~Hz}, 1 \mathrm{H}, \mathrm{H}-2$ '), $8.01(\mathrm{~d}, J=9.2 \mathrm{~Hz}$, 1H, H-8'), 7.35 (m, 3H, H-3' and H-5' and H-7'), 6.51 (d, J=6.5 Hz, 1H, H-9), 3.95 (s, 3H, $\mathrm{OC}_{3}$ ), 3.33 (m, 2H, H-2a and H-8), 3.03 (m, 1H, H-2b), 2.81-2.65 (m, 3H, H-3 and H-6), 2.33 (m, 1H, H-4), 2.18 (s, 3H, H-11), 2.15 (s, 3H, H-13), 1.77 (m, 2H, H-7), 1.49-1.34 (m, 4H, H-5). ${ }^{13} \mathrm{C}$ NMR (75 MHz, $\mathrm{CDCl}_{3}$ ) $\delta$ (ppm): 209.2 (C-10), 169.9 (C-12), 158.0 (C-6'), 147.4 (C-2'), 144.7 (C-10'), 143.4 (C-4'), 131.8 (C-8'), 126.8 (C-9'), 121.8 (C-7’), 118.5 (C3'), 101.4 (C-5'), 73.8 (C-9), 58.4 (C-8), $55.7\left(\mathrm{OCH}_{3}\right), 50.2$ (C-3), 49.5 (C-6), 43.4 (C-2), 29.5 (C-7), 28.9 (C-11), 25.4 (C-4), 21.2 (C-5), 21.1 (C-13). HRMS ( $\left.\mathrm{C}_{22} \mathrm{H}_{26} \mathrm{~N}_{2} \mathrm{O}_{4}\right)$ : Calcd. 382.18926, Found 382.1906. m.p. $59^{\circ} \mathrm{C}$. $[\alpha]_{\mathrm{D}}: 35^{\circ}\left(c 0.56, \mathrm{CHCl}_{3}, 20^{\circ} \mathrm{C}\right)$.

\section{(3S)-O-Acetyl-10,10-difluoro-10,11-dihydro-quinidine 28}

Starting material 24b (105 mg, $0.29 \mathrm{mmol}) ; \mathrm{HF} / \mathrm{SbF}_{5} \mathrm{v} / \mathrm{v}: 3 / 1.5 \mathrm{~mL}$; Temperature: $-40^{\circ} \mathrm{C}$; Reaction time: 10 min., Flash chromatography over $\mathrm{SiO}_{2}$ : EtOAc/Et $2 \mathrm{NH}$ : 98/2, Yield for 28: (105 mg, 91\%, colourless oil). The spectral data for 28 were as follows: ${ }^{1} \mathrm{H}$ NMR $(300 \mathrm{MHz}$, $\left.\mathrm{CDCl}_{3}\right) \delta(\mathrm{ppm}): 8.72\left(\mathrm{~d}, J=4.6 \mathrm{~Hz}, 1 \mathrm{H}, \mathrm{H}-2^{\prime}\right), 8.01$ (d, $\left.J=9.2 \mathrm{~Hz}, 1 \mathrm{H}, \mathrm{H}-8^{\prime}\right), 7.47$ (d, $J=$ $4.5 \mathrm{~Hz}, 1 \mathrm{H}, \mathrm{H}-5$ ') 7.37 (dd, $J=4.5 \mathrm{~Hz}, J=9.2 \mathrm{~Hz}, 1 \mathrm{H}, \mathrm{H}_{-1}$ '), 7.33 (d, $J=4.5 \mathrm{~Hz}, 1 \mathrm{H}, \mathrm{H}-3$ '), 
$6.68(\mathrm{~d}, J=4.3 \mathrm{~Hz}, 1 \mathrm{H}, \mathrm{H}-9), 3.97$ (s, 3H, $\left.\mathrm{OC}_{3}\right), 3.40$ (m, 1H, H-2a), 3.30 (m, 1H, H-8), 3.01 (m, 2H, H-2b and H-6a), 2.83 (m, 1H, H-6b), 2.24 (m, 2H, H-4 and H-7a), 2.15 (s, 3H, H-13), 1.99 (m, 1H, H-3), 1.64 (t, $J=18.6 \mathrm{~Hz}, 3 \mathrm{H}, \mathrm{H}-11), 1.52$ (m, 3H, H-5 and H-7b). ${ }^{13} \mathrm{C}$ NMR (75 MHz, $\left.\mathrm{CDCl}_{3}\right) \delta(\mathrm{ppm}): 170.3$ (C-12), 158.6 (C-6'), 147.6 (C-2'), 145.0 (C-4'), 143.6 (C-10'), 132.1 (C-8’), 127.1 (C-9'), 125.7 (t, $\left.{ }^{1} J_{C F}=223 \mathrm{~Hz}, \mathrm{C}-10\right), 122.6$ (C-3’), 118.7 (C-7'), 101.4 (C-5'), 73.3 (C-9), 58.9 (C-8), $56.1\left(\mathrm{OCH}_{3}\right), 49.9$ (C-6), 44.2 (C-2), 42.4 (t, ${ }^{2} J_{C F}$ $=23.1 \mathrm{~Hz}, \mathrm{C}-3), 27.1(\mathrm{C}-5), 23.8(\mathrm{C}-7), 23.3\left(\mathrm{t},{ }^{2} J_{C F}=27.9 \mathrm{~Hz}, \mathrm{C}-11\right), 23.1(\mathrm{C}-4), 21.3(\mathrm{C}-$ 13). ${ }^{19} \mathrm{~F} \mathrm{NMR}\left(282 \mathrm{MHz}, \mathrm{CDCl}_{3}\right) \delta(\mathrm{ppm}):-96.5(\mathrm{~m}) .[\alpha]_{\mathrm{D}}: 68^{\circ}\left(c 1.2, \mathrm{CH}_{2} \mathrm{Cl}_{2}, 20^{\circ} \mathrm{C}\right)$. 
${ }^{1}$ H NMR 2-(4,4-Difluoro-pentyl)-isoindole-1,3-dione 10<smiles>CC(F)(F)CCCN1C(=O)c2ccccc2C1=O</smiles>

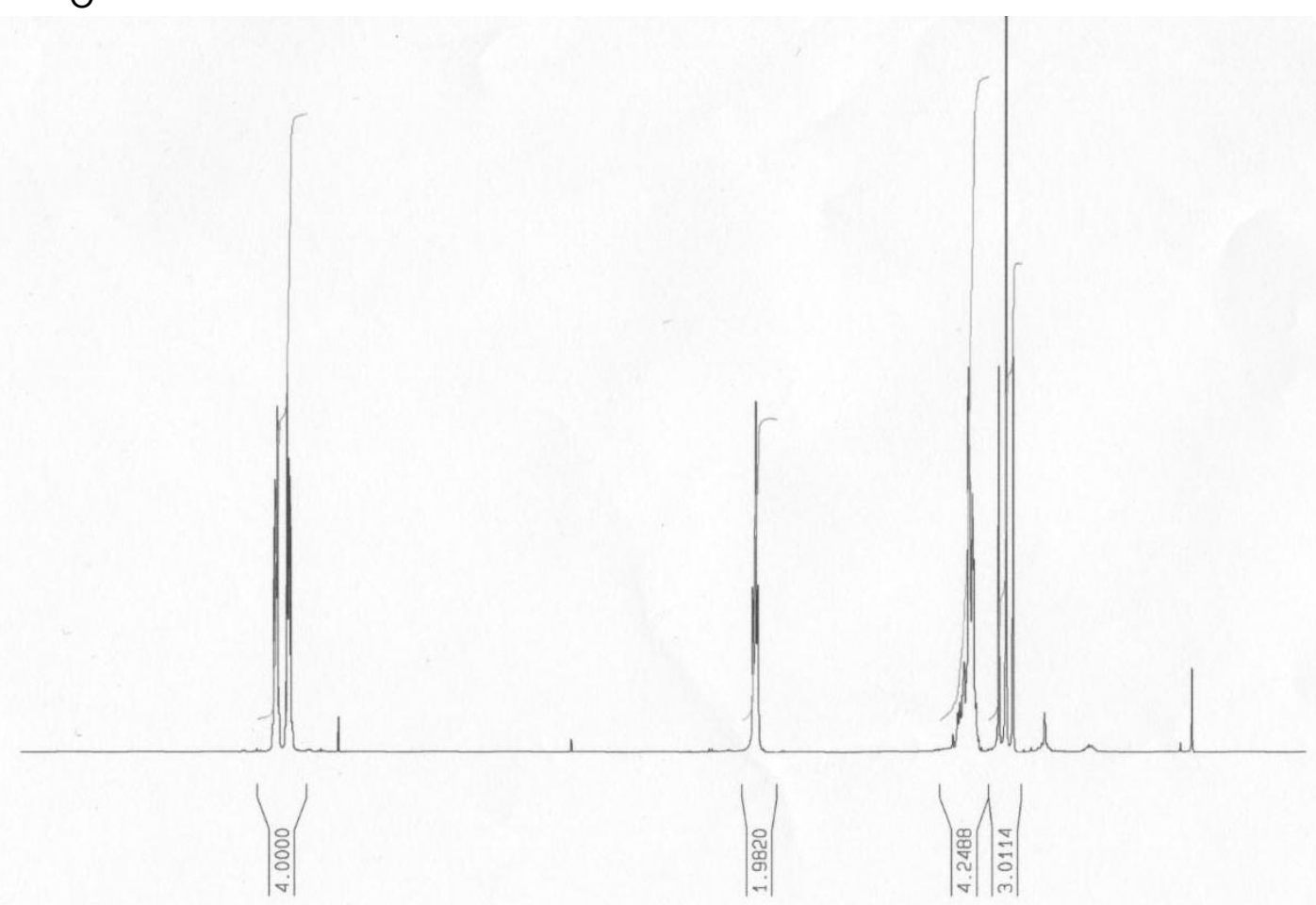

${ }^{19}$ F NMR 2-(4,4-Difluoro-pentyl)-isoindole-1,3-dione 10 
${ }^{1}$ H NMR 2-(4-Oxo-pentyl)-isoindole-1,3-dione 11<smiles>CC(=O)CCCN1C(=O)c2ccccc2C1=O</smiles>

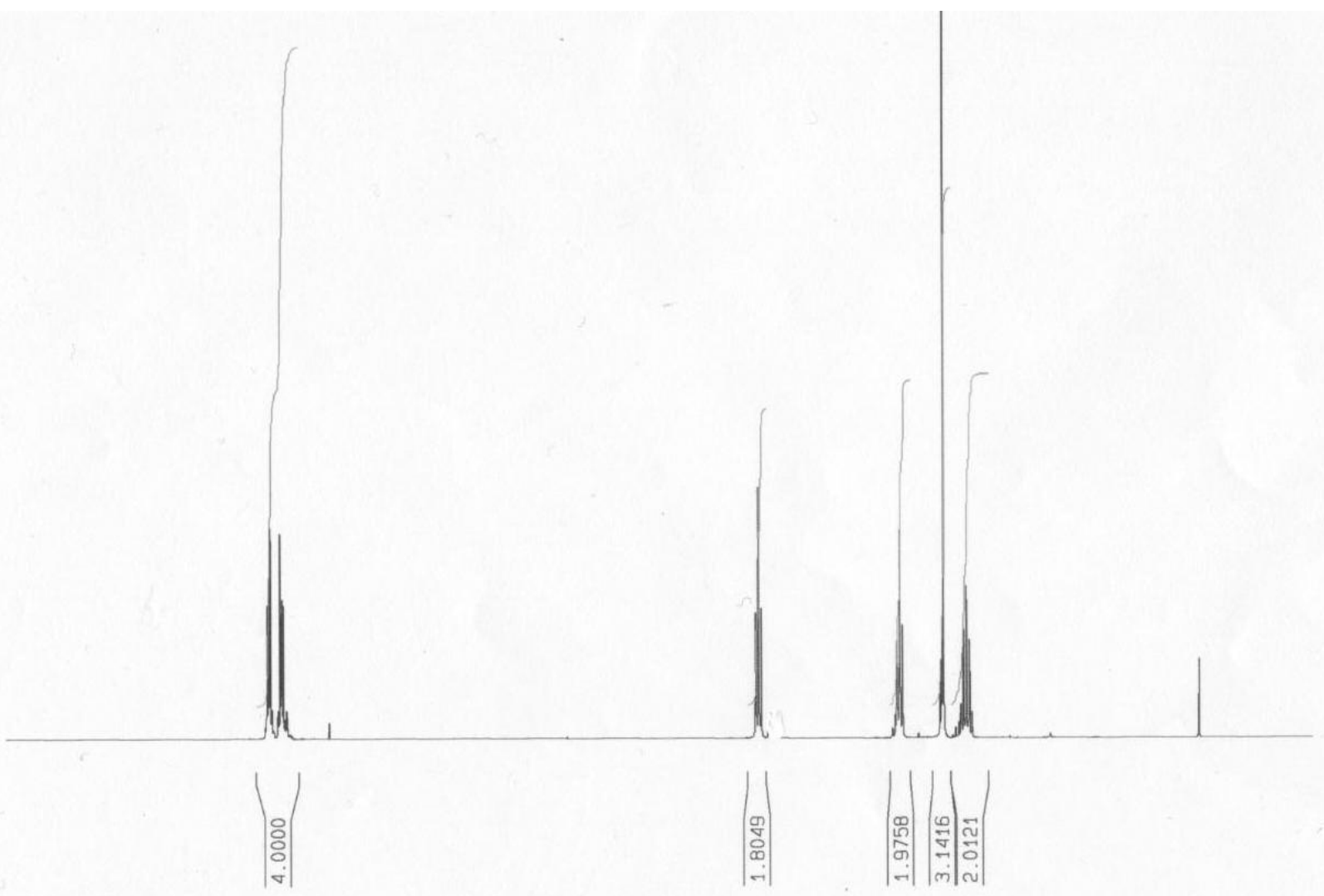


${ }^{1}$ H NMR 1-(4,4-Difluoro-pentyl)-4-phényl-piperidine 12
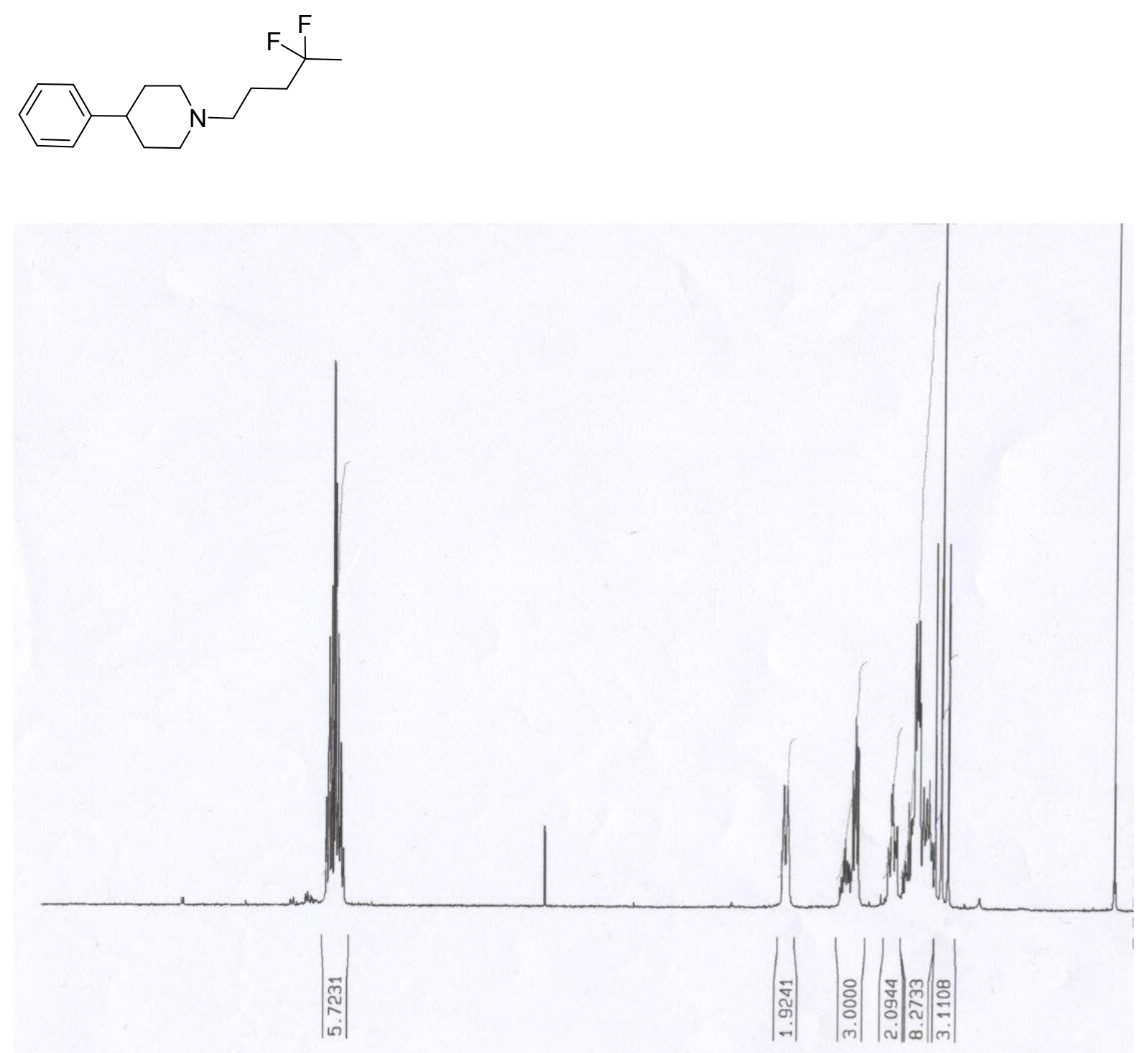
${ }^{13}$ C NMR 1-(4,4-Difluoro-pentyl)-4-phényl-piperidine 12

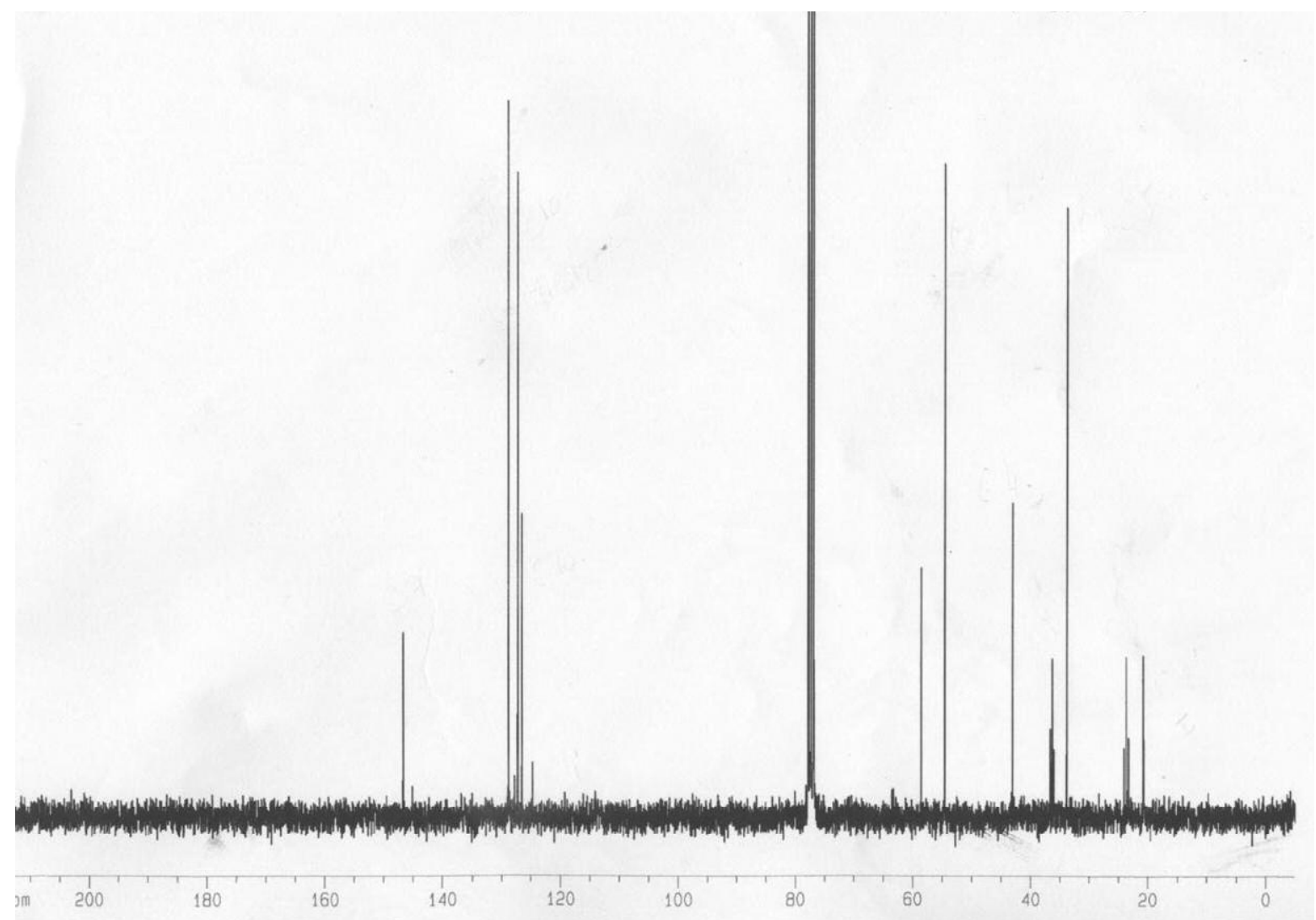


${ }^{1}$ H NMR 2,2-Difluoropropylamine 14

$\mathrm{HCl} . \mathrm{H}_{2} \mathrm{~N} \mathrm{Y}^{\mathrm{F}}$

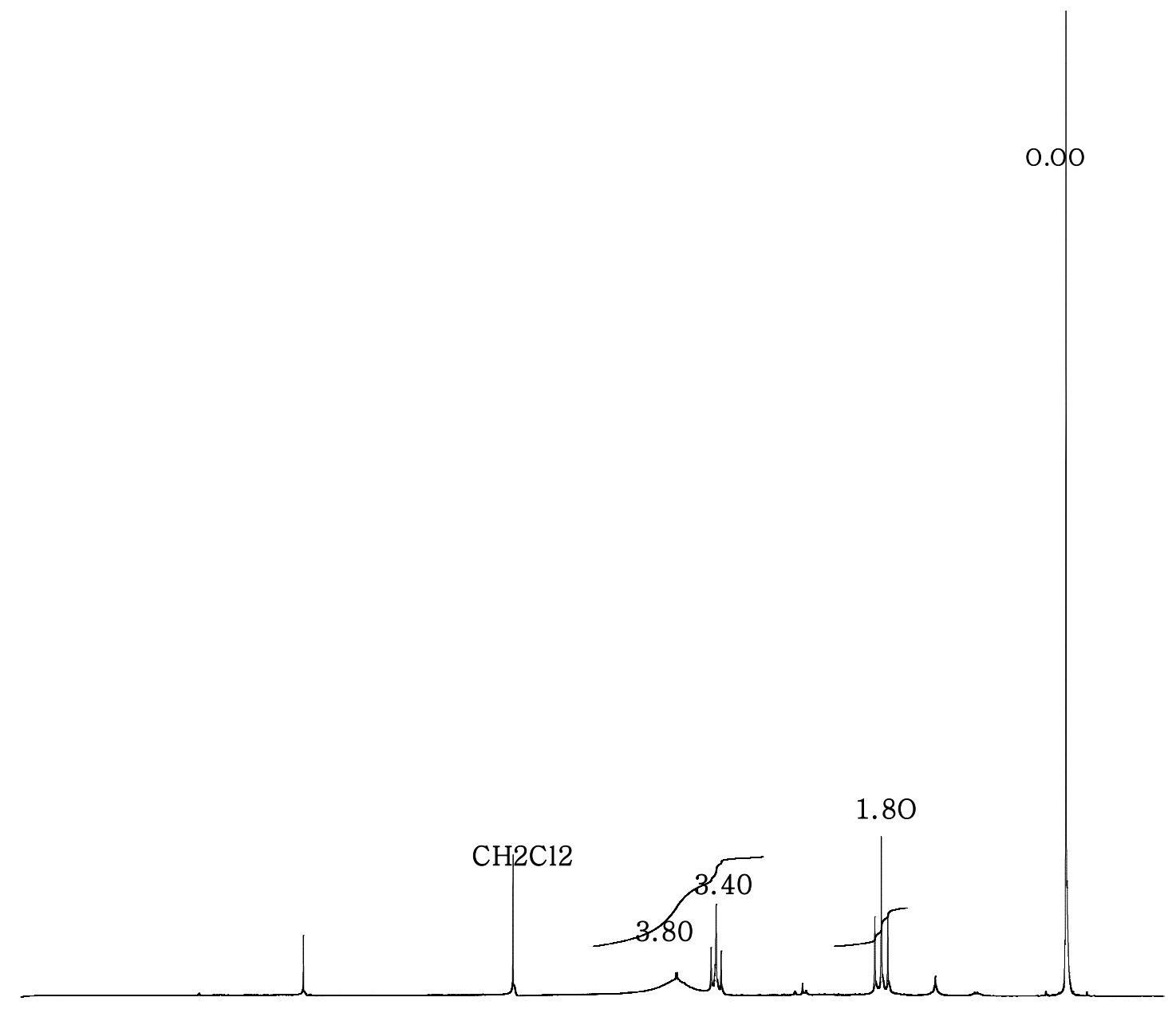

\begin{tabular}{l|l|l|l|l|}
\hline & & \\
$\operatorname{ppm}(\mathrm{t} 1)$ & 5.0 & 0.0
\end{tabular}



ERROR: invalidrestore

OFFENDING COMIMAND: - -restore--

STACK :

- savelevel -

4211

( $\mathrm{S})$

( $\mathrm{s})$

4210

(r)

$(r)$

4209

( $\mathrm{q})$

( $\mathrm{q})$

4208

( $\mathrm{p})$

( $\mathrm{p})$

4207

( 0 )

( 0 )

4206

( $\mathrm{n}$ )

( $\mathrm{n}$ )

4205

( $\mathrm{m}$ )

( $\mathrm{m}$ )

4204

( 1$)$

( 1 )

4203

( $\mathrm{k}$ )

( $\mathrm{k}$ )

4202

( j)

(])

4201

(i)

(i)

4200

( h)

( h)

4199

( $\mathrm{g}$ )

( g)

4198

(f)

( f )

4197

( e)

( e)

4196

(d)

(d)

4195

( C)

( C)

4194

( b)

( b)

4193

( a)

(a)

4192

(')

(')

4191

(-)

( $)$

4190

$(\hat{\wedge})$ 